\title{
PET/MR and PET/CT in a severe COVID-19 patient
}

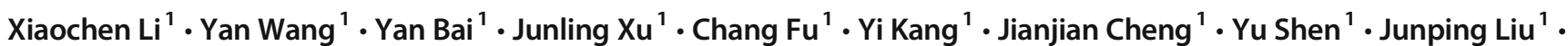 Hewen $\mathrm{Wu}^{1} \cdot$ Weifeng Zhang ${ }^{1} \cdot$ Huiqiang $\mathrm{Li}^{1} \cdot$ Pengyu $\mathrm{Li}^{1} \cdot$ Jianqin $\mathrm{Gu}^{1} \cdot$ Fengmin Shao ${ }^{1} \cdot$ Meiyun Wang ${ }^{1}$}

Received: 4 May 2020 / Accepted: 21 May 2020 / Published online: 4 June 2020

(C) Springer-Verlag GmbH Germany, part of Springer Nature 2020

The clinical manifestations of COVID-19 including cardiovascular dysfunction [1] and impact on other organs have been previously reported [2-4]. However, previous work focused on laboratory indicators to in-directly assess possible damages. PET has potential to identify the spatial distribution of organ dysfunction and could elicit the underlying mechanisms of action. Herein, we report the first imaging manifestations of a severe COVID-19 patient using PET/MR. The patient was also scanned using $\mathrm{PET} / \mathrm{CT}$ in order to provide confirmation of the PET findings, especially given the paucity of reports using PET/CT in COVID-19 [5].

A 57-year-old man with positive SARS-CoV-2 nucleic acid test was enrolled. The CT scan on the day of admission indicated ground-glass opacities (Fig. 1a-b). He responded poorly to symptomatic treatment which lasted 8 days while chest tightness and palpitation worsened. No history of cardiovascular disease was reported. The patient underwent $\left[{ }^{18} \mathrm{~F}\right] \mathrm{FDG}$ PET/CT. The CT and PET/CT fusion images indicated mild focal ground-glass opacities and bands in both lungs without increased metabolic activity, suggesting the diagnosis of absorption period of COVID-19 (Fig. 1c). CT and PET/CT fusion images revealed normal-sized lymph nodes in the mediastinum but

This article is part of the Topical Collection on Image of the month

Xiaochen Li, Yan Wang, Yan Bai, and Junling Xu contributed equally to the work as first authors.

Jianqin Gu, Fengmin Shao, and Meiyun Wang contributed equally to the work as senior authors.

Meiyun Wang

mywang@ha.edu.cn

1 Henan Provincial People's Hospital, Zhengzhou University People's Hospital, No. 7, Weiwu Road, Zhengzhou 450003, Henan, China with increased metabolic activity, suggesting the presence of inflammatory lymph nodes (Fig. 1d).

In order to investigate the cause of palpitations and its systemic impact on other organs, different MR sequences were used, and a $\left[{ }^{18}\right.$ F $]$ FDG PET/MR scan performed. The cine sequence showed that the size of the left ventricle and the wall thickness were in the normal range, and the systolic motion of left ventricle and the wall thickness ratio were normal (Fig. 2a-b). The cardiac T2-weighted MR imaging demonstrated no myocardial edema, and late gadolinium enhanced images did not demonstrate any pathologies (Fig. 2c-d). The PET images showed no increased FDG uptake (Fig. 2e-f). MR and PET scans of other organs yielded similar results with no remarkable changes in the anatomy and metabolism of the brain and spleen (Fig. 3a-f). As for the liver, MR images indicated no remarkable changes in anatomy while PET images showed diffuse increase in FDG uptake (Fig. 3c-f).

The increase in $\left[{ }^{18} \mathrm{~F}\right] \mathrm{FDG}$ uptake in the liver and lymph nodes indicated an inflammatory response in these tissues. The absence of cardiovascular effects suggests that the distribution of angiotensin-converting enzyme 2 and the organs' damage are possibly not correlated in this case. Details about individual differences as well as mechanism require further investigation and longer-term follow-up of the patient.

Author contributions Dr. M-Wang had full access to all the data in the study and takes responsibility for the integrity of the data and the accuracy of the data analysis.

\section{Compliance with ethical standards}

Conflict of interest The authors declare that they have no conflict of interest.

Ethical approval This study was approved by the ethics committee of the Henan Provincial People's Hospital. 

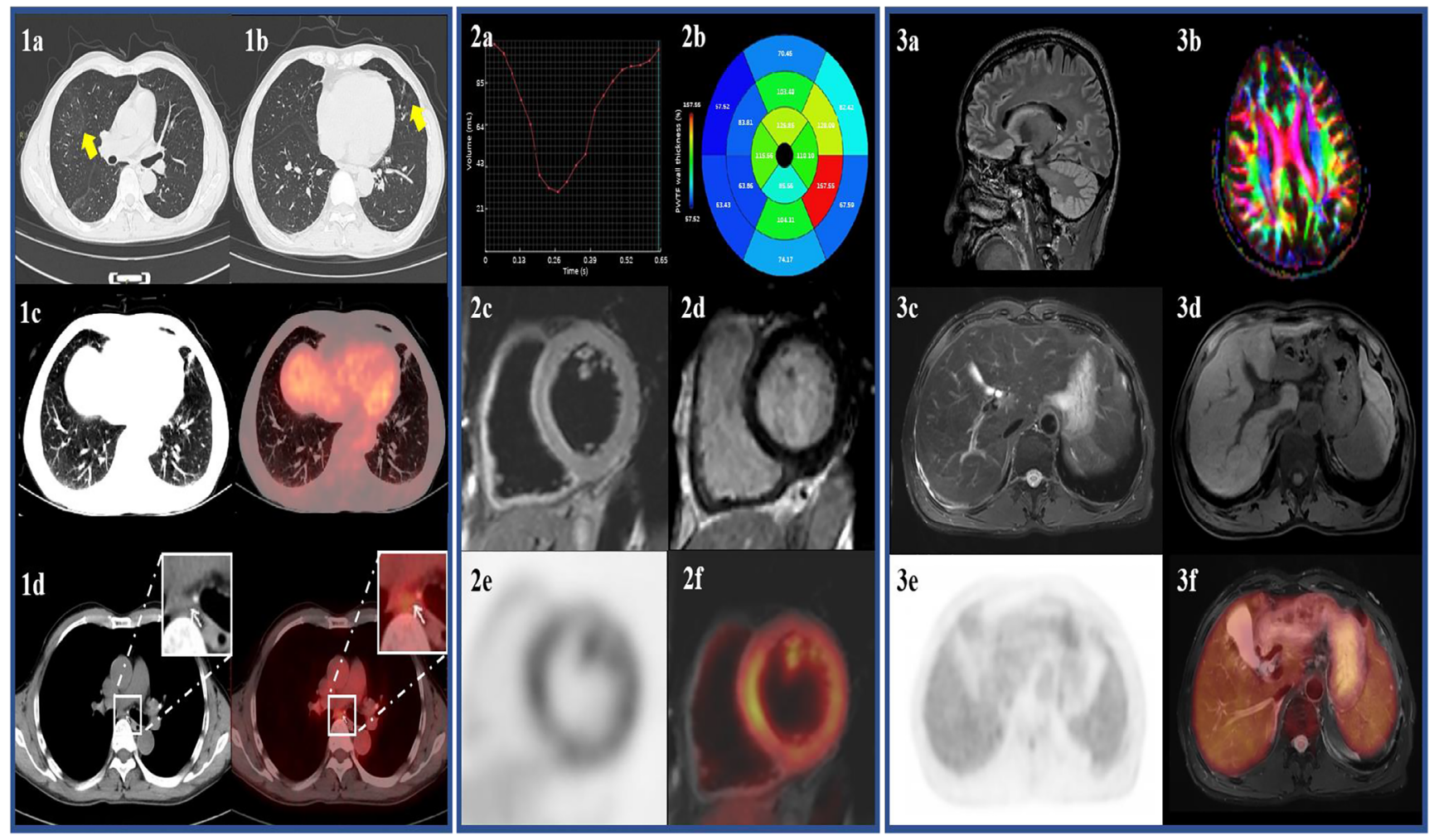

Informed consent Informed consent was obtained from the patient for the anonymous use of the clinical, imaging, and histologic data for publication.

\section{References}

1. Huang C, Wang Y, Li X, et al. Clinical features of patients infected with 2019 novel coronavirus in Wuhan, China. Lancet. 2020;395(10223):497-506.

2. Zhang C, Shi L, Wang F-S. Liver injury in COVID-19: management and challenges. Lancet Gastroenterol Hepatol. 2020. in press. https:// doi.org/10.1016/S2468-1253(20)30057-1(2020).
3. Poyiadji N, Shahin G, Noujaim D, et al. COVID-19-associated acute hemorrhagic necrotizing encephalopathy: CT and MRI features. Radiology. 2020. in press. https://doi.org/10.1148/radiol. 2020201187.

4. Ma L, Xie W, Li D, et al. Effect of SARS-CoV-2 infection upon male gonadal function: a single center-based study. medRxiv. 2020. https://doi.org/10.1101/2020.03.21.20037267.

5. Qin C, Liu F, Yen T-C, et al. ${ }^{18}$ F-FDG PET/CT findings of COVID19: a series of four highly suspected cases. Eur J Nucl Med Mol Imaging. 2020;47(5):1281-6.

Publisher's note Springer Nature remains neutral with regard to jurisdictional claims in published maps and institutional affiliations. 